\title{
Quadruplex-Coupled Kinetics Distinguishes Ligand Binding Between G4 DNA Motifs
}

\author{
Kangkan Halder and Shantanu Chowdhury*
}

Principle and Theoretical Aspects of Surface Plasmon Resonance Analysis

Surface Plasmon Resonance (SPR) biosensor is a label free real-time optical detector and can be used for determination of kinetic rate constants for any bimolecular reaction. The theoretical aspects and principle behind determination of kinetic rate constants is discussed elaborately by O'Shannessy et. al. (1).

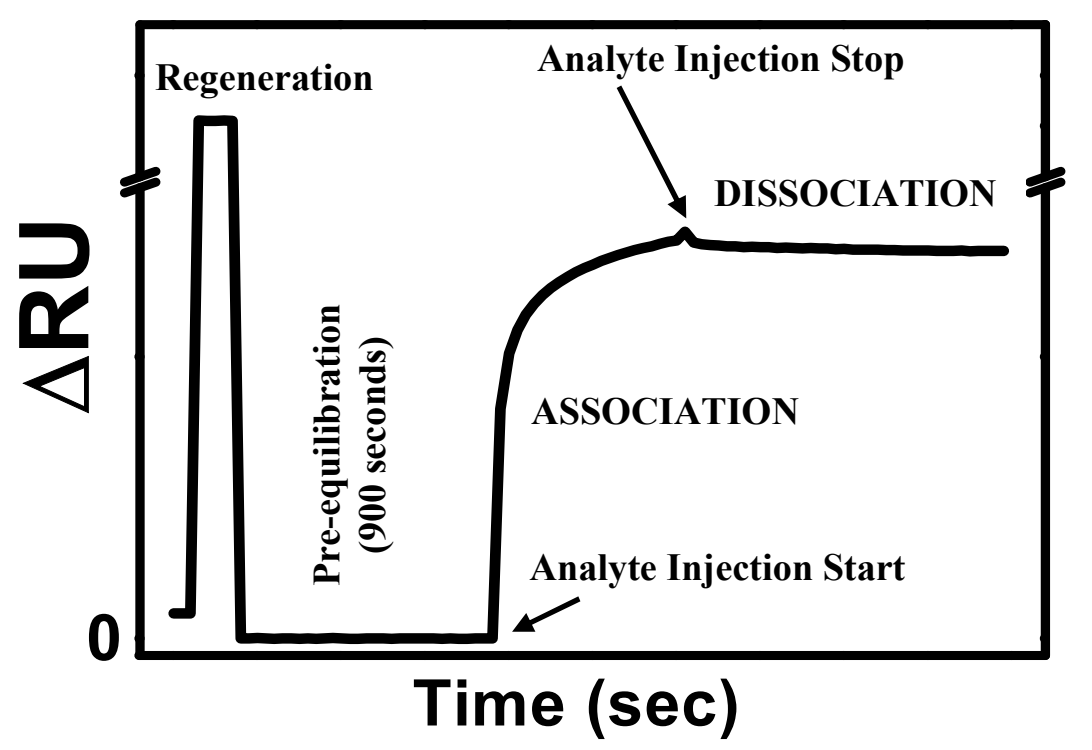

Supplementary Figure 1. Binding of immobilized ligand to analyte molecules results in increase of RU (association) during injection followed by decrease in RU (dissociation) after injection stop. The sensor surface is re-activated by dissociating ligandanalyte complexes (regeneration). This is followed by a pre-equilibration phase to establish the initial equilibrium of sensorattached oligonucleotide molecules. 


\section{Quadruplex-Coupled Ligand (QCL) binding model}

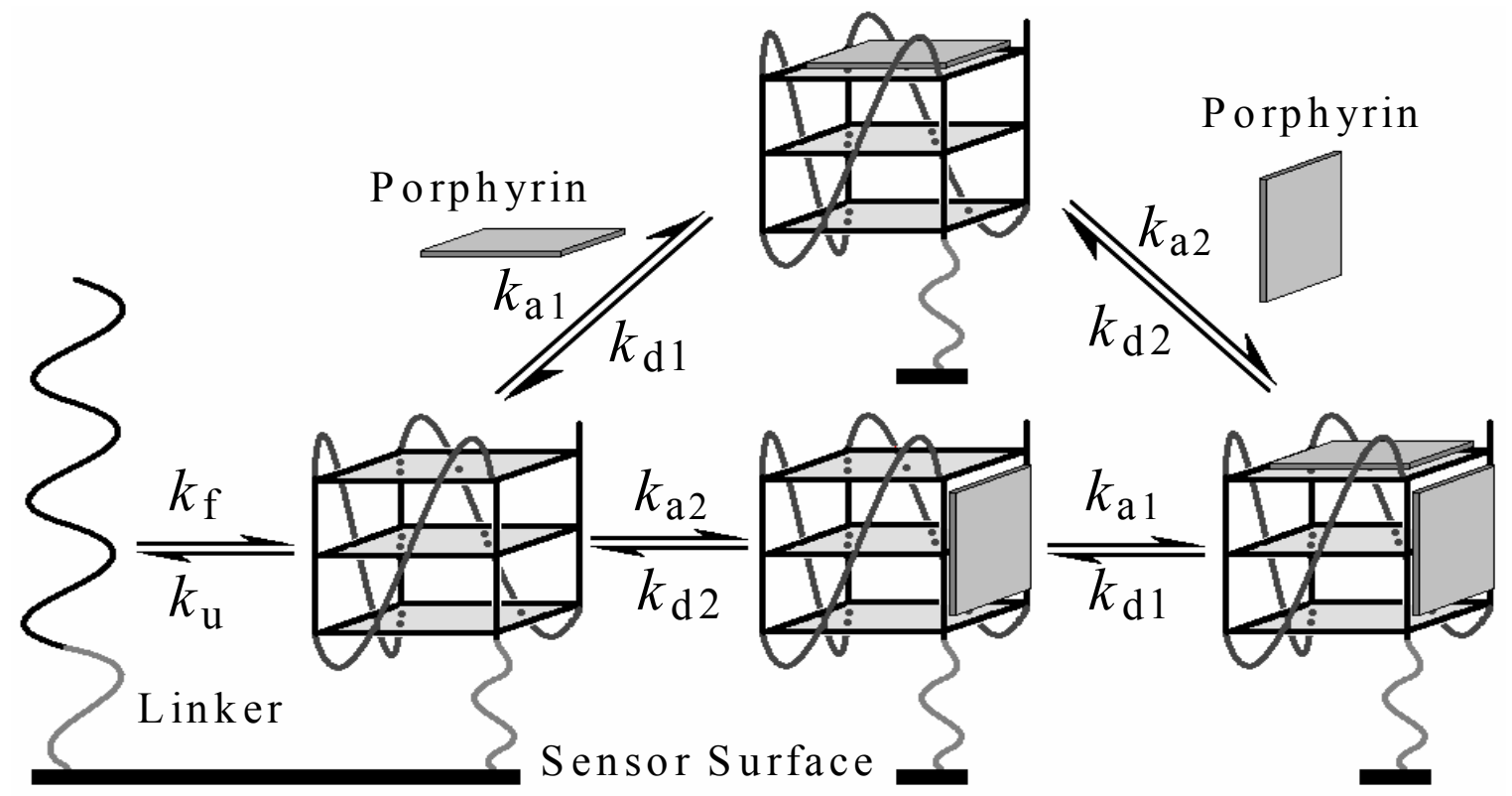

Supplementary Figure 2. Schematic showing folded G-quadruplex molecules immobilized on sensor surface interacting with porphyrin. $k_{\mathrm{u}}$ and $k_{\mathrm{f}}$ represent the inherent unfolding/folding equilibrium of the G-rich oligonucleotide attached to sensor surface using a linker (9-mer spacer oligonucleotide), which is coupled to the subsequent porphyrin binding. The porphyrin interaction model used to solve quadruplex-coupled ligand binding is shown as two mutually exclusive binding interactions represented by respective binding constants. Only the folded G-quadruplex molecule is suitable for porphyrin binding. Ligand interactions showing stacking (binding mode 1) and groove-binding (binding mode 2) are based on several models discussed in literature (2-5).

The quadruplex-coupled ligand (QCL) binding model is discussed considering the several phases of the sensorgram as shown in Supplementary Figure 1.

\section{REGENERATION}

Regeneration buffer is a high salt and high $\mathrm{pH}$ solution, which disintegrates all ligand-analyte complex formed on chip surface.

\section{PRE-EQUILIBRIUM}

After regeneration, the sensor surface was exposed to running buffer for another $900 \mathrm{sec}^{\S}$ before starting analyte injection, as shown Supplementary Figure 1. During this period, the surface immobilized G- rich oligonucleotide attainsequilibrium between the two possible structural isoforms, one being the folded conformation $(F)$ representing the G-quadruplex, while the other represents the unstructured conformation $(U)$. The kinetic expression for the above equilibrium would thus be,

\begin{tabular}{|c|c|}
\hline & $F$ \\
\hline at time, $t=0$ & $R_{\text {max }}$ \\
\hline at time, $t=t_{\mathrm{e}}$, & $F_{\mathrm{e}}$ \\
\hline
\end{tabular}

where, $k_{\mathrm{u}}$ and $k_{\mathrm{f}}$ are unfolding and folding rate constants of the immobilized oligonucleotide, $R_{\max }$ is the concentration of total immobilized ligand on chip surface and $F_{\mathrm{e}}$ is the concentration of folded conformation at any time $t=t_{\mathrm{e}}$, which would be available for ligand (small molecule in mobile phase) association at the initiation of ligand injection. The

$\S$ Earlier, we had determined the folding-unfolding rates for the 31-mer $c$-MYC G-quadruplex. Here, we determined this for all the five sequences and found that all of them reach equilibrium well within 900 seconds. 
high monovalent ion concentration used during regeneration $(1 \mathrm{M} \mathrm{KCl})$ ensures maximal folded structures whereby at time $t=0, U$ has been taken to be 0 . The differential rate expression of the above equation can be written as,

$$
\frac{d F}{d t}=k_{\mathrm{f}}\left(R_{\max }-F\right)-k_{\mathrm{u}} F
$$

where, $F$ is the concentration of the folded isoform formed at any time $t$. Now, under equilibrium conditions, the above expression on integration would give

$$
F_{\mathrm{e}}=\frac{k_{\mathrm{f}} R_{\max }}{\left(k_{\mathrm{u}}+k_{\mathrm{f}}\right)}
$$

where, $F_{\mathrm{e}}$ is the amount of folded species under equilibrium condition. Hence, the unfolded species left $\left(U_{\mathrm{e}}\right)$ on the surface would be,

$$
U_{\mathrm{e}}=\frac{k_{\mathrm{u}} R_{\max }}{\left(k_{\mathrm{u}}+k_{\mathrm{f}}\right)}
$$

The unfolded amount of the oligonucleotide would generate folded species as and when the above equilibrium is disturbed.

\section{ASSOCIATION PHASE}

The association phase is defined as part of the sensorgram between analyte injection start and stop. It represents the formation of the ligand-analyte complex in real time. Here we consider that the small molecule ligand, porphyrin is interacting with the folded quadruplex and not with single-stranded unstructured sequence (Supplementary Figure 2). Also, two independent modes of interaction were considered (sequential binding or the conformation change model as given by manufacturer in BIAevaluation 3.1.1. showed poor fitting to sensorgrams, discussed). Hence the kinetic model can be represented as,

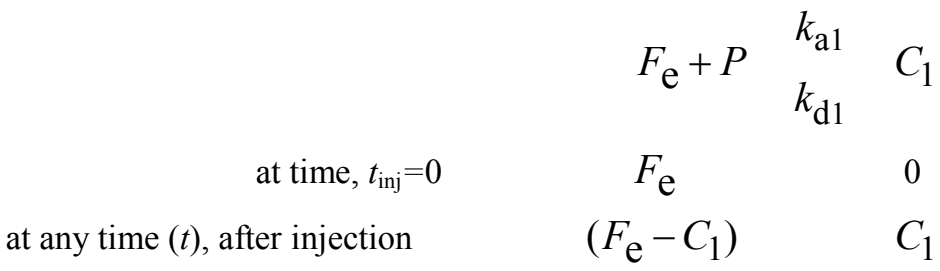

similarly, for the second interaction mode we have,

$$
F_{\mathrm{e}}+P \quad \begin{aligned}
& k_{\mathrm{a} 2} \\
& k_{\mathrm{d} 2}
\end{aligned} C_{2}
$$

at time, $t_{\mathrm{inj}}=0$

at any time $(t)$, after injection

$$
F_{\mathrm{e}} \quad 0
$$

$$
\left(F_{\mathrm{e}}-C_{2}\right) \quad C_{2}
$$

where, $P$ is the concentration of the porphyrin (in injection buffer) but binding to two independent sites of a G-quadruplex, $F_{\mathrm{e}}$ is the amount of preformed folded species present on the sensor surface and is defined in Equation (3), $C_{1}$ and $C_{2}$ are the complexes formed due to the two independent interactions with $k_{\mathrm{a} 1}$ and $k_{\mathrm{d} 1}$ as the association and dissociation rate constants for the first mode (Equation 5) and $k_{\mathrm{a} 2}$ and $k_{\mathrm{d} 2}$ are the association and dissociation rate constants for the second mode (Equation 6), respectively.

Mathematically, Equation 5 can be expressed as,

$$
\frac{d C_{1}}{d t}=k_{\mathrm{a} 1} P\left(F_{\mathrm{e}}-C_{1}\right)-k_{\mathrm{d} 1} C_{1}
$$


On integration, we have,

$$
C_{1}=\left\{\frac{P k_{\mathrm{a} 1} F_{\mathrm{e}}\left(1-e^{-\left(\left(P k_{\mathrm{a} 1}+k_{\mathrm{d} 1}\right)\left(t-t_{0}\right)\right)}\right)}{\left(P k_{\mathrm{a} 1}+k_{\mathrm{d} 1}\right)}\right\}
$$

where ' $t-t_{0}$ ' represents the time elapsed after injection start.

Substituting for $F_{\mathrm{e}}$ from equation (3) we have,

$$
C_{1}=\left\{\frac{P k_{\mathrm{a} 1} k_{\mathrm{f}} R_{\max }\left(1-e^{-\left(\left(P k_{\mathrm{a} 1}+k_{\mathrm{d} 1}\right)\left(t-t_{0}\right)\right)}\right)}{\left(\left(P k_{\mathrm{a} 1}+k_{\mathrm{d} 1}\right)\left(k_{\mathrm{u}}+k_{\mathrm{f}}\right)\right)}\right\}
$$

Similarly, for equation (6), the rate law expression would be,

$$
C_{2}=\left\{\frac{P k_{\mathrm{a} 2} k_{\mathrm{f}} R_{\max }\left(1-e^{-\left(\left(P k_{\mathrm{a} 2}+k_{\mathrm{d} 2}\right)\left(t-t_{0}\right)\right)}\right)}{\left(\left(P k_{\mathrm{a} 2}+k_{\mathrm{d} 2}\right)\left(k_{\mathrm{u}}+k_{\mathrm{f}}\right)\right)}\right\}
$$

where, $R_{\max }$ is the maximum amount of response expected from the complete interaction of porphyrin $P$ and the folded quadruplex $F_{\mathrm{e}}$. It must be noted that in the above set of expressions both binding modes interact with the same quadruplex in two independent sites and are mutually exclusive. The response (RU) generated due to the interaction is dependent on the rate of change in mass only on the sensor surface. Thus the total response generated due to both the interaction modes with the preformed folded conformation is the summation of $C_{1}$ and $C_{2}$.

Due to the above binding interactions with the folded oligonucleotide, the primary equilibrium on the sensor surface is disturbed and hence the remaining unfolded G-rich sequences $\left(U_{\mathrm{e}}\right)$ on the sensor surface would fold to form the quadruplex $\left(F_{\mathrm{t}}\right)$ during the injection time and can be expressed as follows,

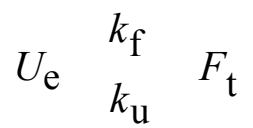

where, $F_{\mathrm{t}}$ is the folded species forming during injection from the pre-equilibrated unfolded isoform $U_{\mathrm{e}}$. Now, the newly formed quadruplex can interact with porphyrin as discussed earlier (Equation 5 and 6). However, folding rate of the oligonucleotide $\left(k_{\mathrm{f}}\right)$ is very slow compared to the rates of association of both the modes of complex formation. Therefore, the rate of folding would be the rate-limiting step and thus the additional ligand-analyte complex $\left(C_{\mathrm{t}}\right)$, resulting from oligonucleotides folding during injection can be expressed as,

$$
\frac{d C_{\mathrm{t}}}{d t}=k_{\mathrm{f}}\left(U_{\mathrm{e}}-C_{\mathrm{t}}\right)
$$

On integration, we have,

$$
C_{\mathrm{t}}=U_{\mathrm{e}}\left(1-e^{-k_{\mathrm{f}}\left(t-t_{0}\right)}\right)
$$

Substituting for $U_{\mathrm{e}}$ from Equation 4,

$$
C_{\mathrm{t}}=\left\{\frac{k_{\mathrm{u}} R_{\max }\left(1-e^{-k_{\mathrm{f}}\left(t-t_{0}\right)}\right)}{\left(k_{\mathrm{u}}+k_{\mathrm{f}}\right)}\right\}
$$

It is interesting to note that the above expression is independent of the porphyrin concentration.

Finally, the real-time response (RU) observed in a quadruplex-coupled-ligand-interaction (QCL) sensorgram can be mathematically expressed as the summation of all the above components and can be expressed as,

$$
R_{\mathrm{t}(\text { assoc })}=C_{1}+C_{2}+C_{\mathrm{t}}+R_{\mathrm{i}}
$$

where, $R_{\mathrm{i}}$ is a fitting parameter which accounts for any change in response (RU) at the time of injection due to any difference between the refractive indices of the running buffer and injection buffer and has been discussed previously $(1 ; 6)$. Therefore, the association phase of the sensorgram can be summarized as, 


$$
\begin{aligned}
R_{\mathrm{t}(\operatorname{assoc})}= & \left\{\frac{P k_{\mathrm{a} 1} k_{\mathrm{f}} R_{\max }\left(1-e^{-\left(\left(P k_{\mathrm{a} 1}+k_{\mathrm{d} 1}\right)\left(t-t_{0}\right)\right)}\right)}{\left(\left(P k_{\mathrm{a} 1}+k_{\mathrm{d} 1}\right)\left(k_{\mathrm{u}}+k_{\mathrm{f}}\right)\right)}\right\}+\left\{\frac{P k_{\mathrm{a} 2} k_{\mathrm{f}} R_{\max }\left(1-e^{-\left(\left(P k_{\mathrm{a} 2}+k_{\mathrm{d} 2}\right)\left(t-t_{0}\right)\right)}\right)}{\left(\left(P k_{\mathrm{a} 2}+k_{\mathrm{d} 2}\right)\left(k_{\mathrm{u}}+k_{\mathrm{f}}\right)\right)}\right\}+ \\
& \left\{\frac{k_{\mathrm{u}} R_{\max }\left(1-e^{-k_{\mathrm{f}}\left(t-t_{0}\right)}\right)}{\left(k_{\mathrm{u}}+k_{\mathrm{f}}\right)}\right\}+R_{\mathrm{i}}
\end{aligned}
$$

\section{Biphasic ligand binding to G-quadruplex using Fluorescence Resonance Energy Transfer}

In order to confirm our SPR data, which indicated two binding sites, we performed independent experiments in solution (unlike the surface-attached kinetics in SPR) using fluorescence resonance energy transfer (FRET) technique. These experiments were performed using $100 \mathrm{nM}$ of the $c-M Y C$ promoter (FmycG27T, Figure 3a) or human telomeric (FhtG21T, Figure 3b) doubly labeled (5'-Fluorescein and 3'-TAMRA, which constitutes a donoracceptor pair) G-quadruplex sequence and was titrated with TmPyP4 or TpPy $(0-10 \mu \mathrm{M})$.

TmPyP4 and TpPy in all cases showed interaction with two binding sites, except in case of the Tetrahymena tetraplex - TpPy interaction (Supplementary Table 1). Interestingly, a single-site binding model was also not adequate, indicating complex binding interactions.

Supplementary Table 1. Equilibrium ligand-G-quadruplex binding constants observed by fluorescence resonance energy transfer (FRET).

\begin{tabular}{cccc}
\hline Ligand-quadruplex pair & $\boldsymbol{K}_{\mathrm{D} 1}{ }^{a}(\mu \mathrm{M})$ & $\boldsymbol{K}_{\mathbf{D} 2}{ }^{a}(\mu \mathrm{M})$ & $\boldsymbol{K}_{\mathrm{D} 1} / \boldsymbol{K}_{\mathbf{D} 2}$ \\
\hline FmycG27T - TmPyP4 & 1.098 & 0.107 & 10.255 \\
FmycG27T - TpPy & 0.334 & 0.083 & 4.029 \\
FhtG21T - TmPyP4 & 3.310 & 0.193 & 17.157 \\
FhtG21T - TpPy & 0.558 & 0.475 & 1.177 \\
FofG28T - TmPyP4 & 6.159 & 0.364 & 16.943 \\
FofG28T - TpPy & 1.278 & 0.359 & 3.560 \\
FttG22T - TmPyP4 & 5.418 & 0.154 & 35.184 \\
FttG22T - TpPy & 1.942 & 0.082 & 23.765 \\
\hline
\end{tabular}

parameters were determined using the Scatchard analysis.

Comparison of kinetic parameters obtained by QCL model with previous G4-ligand binding SPR results

Supplementary Table 2. Equilibrium and kinetic constants of G-quadruplex binding to small molecule ligands determined by SPR in previous studies ${ }^{a}$

\begin{tabular}{cccccc}
\hline G-quadruplex & Ligand & $\boldsymbol{K}_{\mathrm{D}}(\mathbf{M})$ & $\boldsymbol{k}_{\mathbf{d}}\left(\mathbf{s}^{-1}\right)$ & $\boldsymbol{k}_{\mathbf{a}}\left(\mathbf{M}^{-1} \mathbf{s}^{-1}\right)$ & Reference \\
\hline C-MYC $^{b}$ & $\mathrm{Se}_{2} \mathrm{SAP}$ & $10^{-7}$ & 0.0029 & $10^{3}$ & $(2)$ \\
Tetrahymena telomeric $^{\mathrm{b}}$ & $\mathrm{Se}_{2} \mathrm{SAP}$ & $10^{-7}$ & 0.0041 & $10^{3}$ & $(2)$ \\
TBA $^{\mathrm{b}}$ & $\mathrm{Se}_{2} \mathrm{SAP}$ & $10^{-7}$ & 0.0094 & $10^{3}$ & $(2)$ \\
human telomeric & Bis(quinacridine) macrocycle & $10^{-7}$ & - & - & $(4)$ \\
human telomeric & 3,6,9-trisubstituted acridine & $10^{-5}-10^{-7}$ & $0.2-0.02$ & $10^{5}-10^{7}$ & $(7)$ \\
human telomeric & TmPyP4 & $10^{-6}$ & 0.0066 & $10^{3}$ & $(8)$ \\
\hline
\end{tabular}

\footnotetext{
${ }^{a}$ blank spaces indicate parameters not determined; ${ }^{b}$ values for the high affinity binding site is shown - the second binding mode is at least 10 -fold lower in affinity. All $k_{\mathrm{a}}$ have been determined from steady-state RU using the $k_{\mathrm{d}}$ determined from dissociation sensorgram. TBA- thrombin binding aptamer.
} 


\section{Earlier results on $c$-MYC G-quadruplex stabilization by telomerase inhibitors}

Comparison of the potential of TmPyP4 for telomerase inhibition versus $c-M Y C$ G-quadruplex binding using cell-based assays indicated marginal difference in the $\mathrm{IC}_{50}$ (concentration for $50 \%$ reduction of electrophoresis gel bands) values ( 0.45 and $0.34 \mu \mathrm{M}$ for telomeric and $c-M Y C$, respectively) (9). However, in a second study, a cell-free competition assay indicated an 8-fold preference of TmPyP4 for $c-M Y C$ G-quadruplex binding relative to the human telomeric sequence (2). Though these results are by far the best assays possible to address the complex issue of telomerase inhibition versus $c-M Y C$ promoter binding the assays may be limited due to the additional complexity of PCR amplification and/or taq polymerase extension.

\section{Quadruplex-coupled ligand binding affects $\boldsymbol{k}_{\mathrm{u}}$ but not $\boldsymbol{k}_{\mathrm{f}}$}

Inherent folding/unfolding constants $\left(k_{\mathrm{f}} / k_{\mathrm{u}}\right)$ of the intramolecular G-quadruplex are coupled to hybridization or ligand binding by the quadruplex-coupled (QC) hybridization and QC-ligand binding models (QCL), respectively. Supplementary Table 3 shows ratio of $k_{\mathrm{u}}$ and $k_{\mathrm{f}}$ determined from hybridization (Table 3 ) and ligand binding reactions (Table 2). The overall average values suggest that the folding constants were largely similar whereas the unfolding constants varied. The difference in unfolding constants was larger in case of TpPy relative to TmPyP4, which is consistent with increased stabilization of the folded G-quadruplexes by TpPy.

Supplementary Table 3. Ratio of $k_{\mathrm{f}}$ and $k_{\mathrm{u}}$ obtained from hybridization and ligand binding by the G-quadruplex coupled models

\begin{tabular}{cccc}
\hline & & $\left(k_{\mathrm{u}}\right.$ hyb / $k_{\mathrm{u}}$ ligand binding $)$ & $\left(k_{\mathrm{f}}\right.$ hyb / $k_{\mathrm{f}}$ ligand binding $)$ \\
\hline \multirow{2}{*}{ mycG27B } & TmPyP4 & 4.77 & 1.52 \\
& TpPy & 14.93 & 1.91 \\
htG21B & TmPyP4 & 2.89 & 1.96 \\
& TpPy & 12.84 & 2.34 \\
\multirow{2}{*}{ ofG28B } & TmPyP4 & 2.08 & 1.67 \\
& TpPy & 6.16 & 0.90 \\
ttG22B & TmPyP4 & 2.11 & 1.32 \\
& TpPy & 3.65 & 0.75 \\
\hline Avg. (std. dev) & & $6.18(4.98)$ & $1.55(0.54)$ \\
\hline
\end{tabular}

\section{Quadruplex coupled hybridization with G-quadruplex from c-MYC promoter and human, Oxytricha and Tetrahymena telomere}

Supplementary Figure 3 shows representative sensorgrams for hybridization of the human telomeric Gquadruplex wherein it is evident that the Quadruplex-Coupled (QC) hybridization model (Supplementary Figure 3a) best represents the hybridization reaction. Considering a simple two-state $(\mathrm{A}+\mathrm{B} \rightarrow \mathrm{C})$ hybridization reaction gives 'poor' fitting (Supplementary Figure 3b). Detailed analysis in this regard with respect to the 31-mer c-MYC Gquadruplex is presented in an earlier report (6). We also confirmed our previous finding with the $c-M Y C$ G-quadruplex using the 27-mer sequence from the $c-M Y C$ promoter, which is considered to be biologically active as a repressor. Additionally, we used another 17-mer fragment from the same region, mycG17B, which forms a single parallel quadruplex, in contrast to multiple possible polymorphic parallel G-quadruplex motifs with the 27 or 31-mer sequence in order to address issues of surface heterogeneity. Results showing mostly less than 5\% error in fitting using the QCmodel were obtained for all the G-quadruplex motifs (Supplementary Figure 3). 


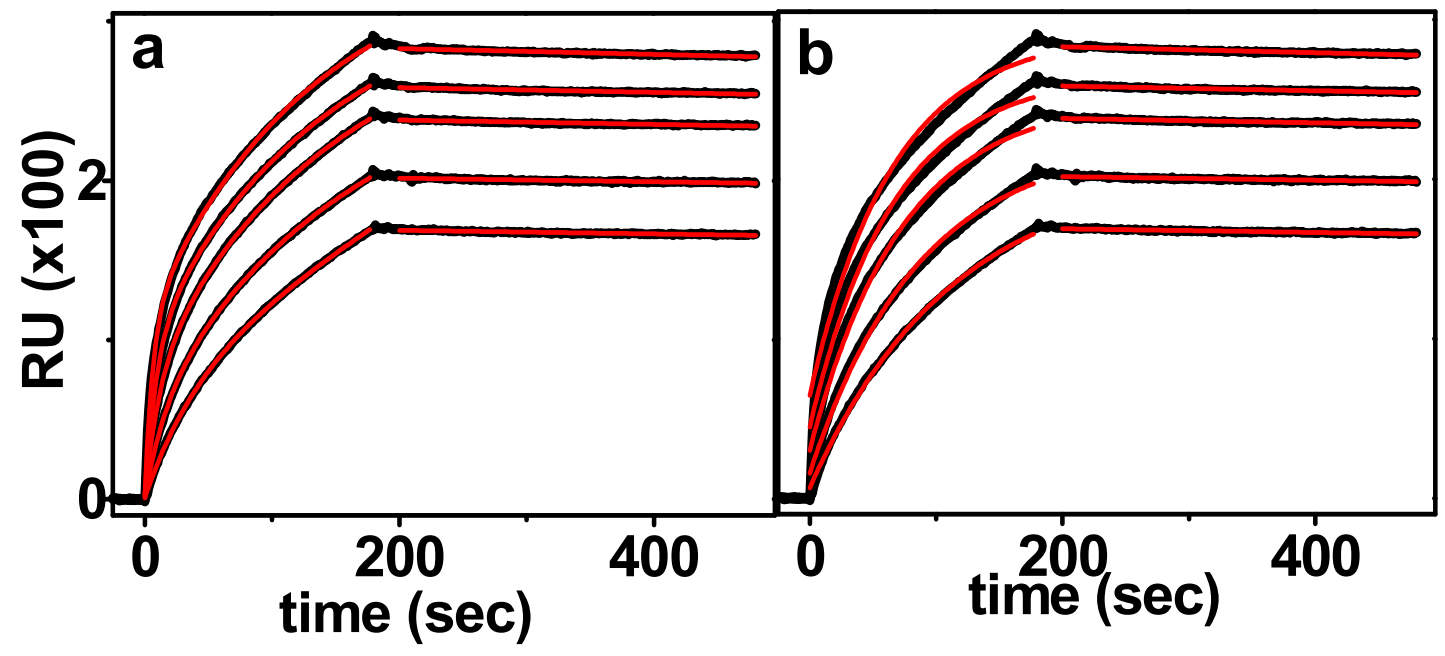

Supplementary Figure 3. Sensorgrams analyzed with quadruplex-coupled versus simple hybridization models. (a, b) Sensorgrams were obtained by hybridization with immobilized htG21B using 64, 128, 256, 512 or $1024 \mathrm{nM}$ of the respective complementary strand (htC21) in the mobile phase. Fitted curves (red) were obtained by fitting the sensorgrams (black) with either (a) the quadruplex-coupled hybridization model ( 6 ) or (b) simple-hybridization model (1:1 Langmuir binding as per BIAevaluation 3.1.1 supplied by manufacturer).

\section{Analysis of ligand-binding versus hybridization competition}

In an intracellular context, ligand binding to G-quadruplex would be subject to competition from inherent hybridization with the complementary strand. It is evident that hybridization rates are high compared to ligand binding rates. However, ligand concentrations may be substantially high relative to the concentration of the complementary strand (which is low and equimolar w.r.t. to the G-quadruplex within a chromosome, particularly for non-telomeric sequences). Therefore, we attempted to resolve these competing equilibria in order to gauge the potential of Gquadruplexes to bind small molecule ligands vis-à-vis duplex formation.

The observed rate of hybridization, $k_{\mathrm{obs}(\mathrm{hyb})}$ may be expressed as,

$$
k_{\mathrm{obs}(\mathrm{hyb})}=k_{\mathrm{a}(\mathrm{hyb})}\left[C_{\mathrm{st}}\right]+k_{\mathrm{d}(\mathrm{hyb})}
$$

where, $k_{\mathrm{a}(\mathrm{hyb})}$ and $k_{\mathrm{d}(\mathrm{hyb})}$ represent association and dissociation rates for hybridization and $C_{\mathrm{st}}$ is concentration of the complementary strand.

Similarly, the corresponding ligand binding rates $k_{\mathrm{obs}(\mathrm{lig})}$ may be expressed as,

$$
k_{\mathrm{obs}(\mathrm{lig})}=k_{\mathrm{a}(\mathrm{lig})}\left[C_{\mathrm{L}}\right]+k_{\mathrm{d}(\mathrm{lig})}
$$

where, $k_{\mathrm{a}(\mathrm{lig})}$ and $k_{\mathrm{d}(\mathrm{lig})}$ represent association and dissociation rates for ligand binding and $C_{\mathrm{L}}$ is concentration of the ligand.

Therefore, the relative rates of hybridization versus ligand binding is,

$$
\frac{k_{\mathrm{obs}(\mathrm{hyb})}}{k_{\mathrm{obs}(\text { lig })}}=\left\{\frac{k_{\mathrm{a}(\mathrm{hyb})}\left[C_{\mathrm{st}}\right]+k_{\mathrm{d}(\mathrm{hyb})}}{k_{\mathrm{a}(\text { lig })}\left[C_{\mathrm{L}}\right]+k_{\mathrm{d}(\text { lig })}}\right\}
$$

Since $k_{\mathrm{a}} \quad k_{\mathrm{d}}$, Equation 19 can be expressed as ratio of the complementary strand and ligand concentrations:

$$
\frac{k_{\mathrm{obs}(\mathrm{hyb})}}{k_{\mathrm{obs}(\mathrm{lig})}}=\left\{\frac{k_{\mathrm{a}(\mathrm{hyb})}\left[C_{\mathrm{st}}\right]}{k_{\mathrm{a}(\mathrm{lig})}\left[C_{\mathrm{L}}\right]}\right\}
$$

We used the SPR sensorgram-derived association constants for hybridization and ligand association to simulate the relative rates for hybridization versus ligand association. This was done at various ratios of 
complementary strand versus ligand concentrations for each ligand-G-quadruplex pair to judge the discriminatory potential of small molecule ligands towards binding in presence of competing duplex formation (Figure 6c).

\section{References}

1. O'Shannessy, D. J., Brigham-Burke, M., Soneson, K. K., Hensley, P., and Brooks, I. (1993) Determination of rate and equilibrium binding constants for macromolecular interactions using surface plasmon resonance: use of nonlinear least squares analysis methods, Anal Biochem 212, 457-468.

2. Seenisamy, J., Bashyam, S., Gokhale, V., Vankayalapati, H., Sun, D., Siddiqui-Jain, A., Streiner, N., Shin-Ya, K., White, E., Wilson, W. D., and Hurley, L. H. (2005) Design and synthesis of an expanded porphyrin that has selectivity for the c-MYC G-quadruplex structure, J Am Chem Soc 127, 2944-2959.

3. Shi, D. F., Wheelhouse, R. T., Sun, D., and Hurley, L. H. (2001) Quadruplex-interactive agents as telomerase inhibitors: synthesis of porphyrins and structure-activity relationship for the inhibition of telomerase, $J$ Med Chem 44, 4509-4523.

4. Teulade-Fichou, M. P., Carrasco, C., Guittat, L., Bailly, C., Alberti, P., Mergny, J. L., David, A., Lehn, J. M., and Wilson, W. D. (2003) Selective recognition of G-qQuadruplex telomeric DNA by a bis(quinacridine) macrocycle, $J$ Am Chem Soc 125, 4732-4740.

5. Yamashita, T., Uno, T., and Ishikawa, Y. (2005) Stabilization of guanine quadruplex DNA by the binding of porphyrins with cationic side arms, Bioorg Med Chem 13, 2423-2430.

6. Halder, K. and Chowdhury, S. (2005) Kinetic resolution of bimolecular hybridization versus intramolecular folding in nucleic acids by surface plasmon resonance: application to G-quadruplex/duplex competition in human c-myc promoter

131, Nucleic Acids Res. 33, 4466-4474.

7. Read, M., Harrison, R. J., Romagnoli, B., Tanious, F. A., Gowan, S. H., Reszka, A. P., Wilson, W. D., Kelland, L. R., and Neidle, S. (2001) Structure-based design of selective and potent G quadruplex-mediated telomerase inhibitors, PNAS 98, 4844-4849.

8. Dixon, I. M., Lopez, F., Tejera, A. M., Esteve, J. P., Blasco, M. A., Pratviel, G., and Meunier, B. (2007) A Gquadruplex ligand with 10000-fold selectivity over duplex DNA, J Am Chem Soc 129, 1502-1503.

9. Lemarteleur, T., Gomez, D., Paterski, R., Mandine, E., Mailliet, P., and Riou, J. F. (2004) Stabilization of the cmyc gene promoter quadruplex by specific ligands' inhibitors of telomerase, Biochem Biophys Res Commun $323,802-808$. 